\title{
Exercício aeróbio: Aspectos bioenergéticos, ajustes fisiológicos, fadiga e índices de desempenho
}

\author{
Aerobic exercise: bioenergetics, physiological \\ adjustments, fatigue and performance indices
}

\author{
Fabrizio Caputo ${ }^{1,2}$ \\ Mariana Fernandes Mendes de Oliveira ${ }^{2}$ \\ Camila Coelho Greco ${ }^{2}$ \\ Benedito Sérgio Denadai ${ }^{2}$
}

1 Universidade para o Desenvolvimento do Estado de Santa Catarina. Centro de Ciências da Saúde e do Esporte. Florianópolis, SC. Brasil.

2 Universidade Estadual Paulista. Laboratório de Avaliação da Performance Humana. Rio Claro, SP. Brasil.

Recebido em 09/12/07 Aprovado em 22/05/08
Resumo - O objetivo deste trabalho foi trazer informações atuais e relevantes a respeito dos aspectos fisiológicos determinantes do rendimento e treinamento dos esportes aeróbios. Diferente do que se tem sido comumente apresentado, o metabolismo aeróbio responde rapidamente a demanda energética, com um exercício máximo de $75 \mathrm{~s}$, apresentando iguais contribuições do sistema aeróbio e anaeróbio para a produção total de energia. No entanto, em exercícios acima dessa duração, os possíveis mecanismos da fadiga relacionados ao metabolismo anaeróbio ainda são os principais determinantes da tolerância ao exercício. Em durações mais prolongadas (acima de $60 \mathrm{~min}$ ), a depleção de glicogênio, o desequilíbrio hídrico e eletrolítico, elevação da temperatura corporal terão um papel decisivo no rendimento. As mais importantes variáveis do desempenho aeróbio têm sido organizadas em um modelo que integra fatores como o consumo máximo de oxigênio $\left(\mathrm{VO}_{2} \mathrm{max}\right)$, limiares relacionados à resposta do lactato ao exercício e a eficiência muscular. Para atletas de elite, observa-se que em adição a um elevado valor de $\mathrm{VO}_{2}$ max, o sucesso em eventos aeróbios também requer uma capacidade de se exercitar por um tempo prolongado a uma alta porcentagem do $\mathrm{VO}_{2} \max$, bem como, uma eficiente conversão da energia produzida para a forma de trabalho muscular. Dependendo da duração da modalidade que se compete, os treinos deverão objetivar uma melhora da $\mathrm{IVO}_{2}$ max, capacidade anaeróbia lática e tolerância à acidose, para as competições mais curtas, capacidade aeróbia para durações intermediárias e a melhora da capacidade de estocar glicogênio e aumentar a utilização de gordura, nas modalidades muito prolongadas. Palavras-chave: Índices fisiológicos, lactato, fadiga, sistemas energéticos.

Abstract - The objective of this study was to present relevant updated information regarding the physiological determinants of aerobic training and performance. In contrast to common concepts, the aerobic metabolism rapidly responds to energy requirements, with the anaerobic and aerobic energy systems equally contributing to total energy production during maximal exercise lasting about $75 \mathrm{~s}$. However, in the case of longer exercise duration the possible mechanisms of fatigue related to anaerobic metabolism are still the main determinants of exercise tolerance. Prolonged exercise (more than one hour) can be limited by several factors such as substrate depletion, water and electrolyte disturbance, or problems related to thermoregulation leading to an increase in body temperature. The most important variables of endurance performance have been organized into a model that integrates factors such as maximum oxygen uptake ( $\mathrm{VO}_{2}$ max), blood lactate thresholds, and muscle efficiency. For highly trained athletes, in addition to a high $\mathrm{VO}_{2} \max$, success in endurance events also requires the ability to exercise for prolonged periods at a high percentage of $\mathrm{VO}_{2} \max$, as well as to efficiently convert the energy produced into muscle work. Depending on the duration of the aerobic event, the training sessions should be aimed at improving $\mathrm{VO}_{2}$ max, anaerobic lactate capacity and acidosis tolerance in the case of short-lasting events and aerobic capacity for events of intermediate duration, and at increasing muscle glycogen content and fat utilization in the case of long-lasting events.

Key words: Physiological indices; Lactate; Fatigue; Energy systems. 


\section{INTRODUÇÃO}

Quais são os limites do rendimento humano? Será possível nadar os $100 \mathrm{~m}$ abaixo de $40 \mathrm{~s}$ ? Será possível correr uma maratona abaixo de 2 horas? Estes seriam objetivos impossíveis, ou eles ainda subestimam os limites do rendimento humano? Estas são questões importantes, de interesse não só de pesquisadores e técnicos, mas também dos atletas e da população em geral. Nos esportes, atletas e técnicos que desejam maximizar o rendimento necessitam de um bom conhecimento dos processos fisiológicos.

Muitas informações referentes aos aspectos que contribuem para o rendimento aeróbio foram obtidas, simplesmente, descrevendo as características fisiológicas de atletas de elite em esportes como, por exemplo, corrida, ciclismo e natação. Os numerosos determinantes do rendimento aeróbio têm sido organizados em um modelo que integra fatores como o consumo máximo de oxigênio $\left(\mathrm{VO}_{2} \max \right)$, limiares relacionados a resposta do lactato sanguíneo ao exercício e a eficiência muscular, todos estes considerados como as mais importantes variáveis do desempenho aeróbio. Para atletas de alto rendimento, observa-se que em adição a um elevado valor de $\mathrm{VO}_{2}$ max, o sucesso em eventos aeróbios também requer uma capacidade de se exercitar por um período de tempo prolongado a uma alta porcentagem do $\mathrm{VO}_{2} \max$, bem como, uma eficiente conversão da energia produzida para a forma de movimento corporal. Com base nas variações que podem ocorrer nesta eficiência ou economia de movimento (EM), é possível entender porque a intensidade de exercício associada ao $\mathrm{VO}_{2} \max \left(\mathrm{IVO}_{2} \max \right)$ pode ser bem diferente entre atletas que possuem valores similares de $\mathrm{VO}_{2}$ max. Genericamente, a $\mathrm{IVO}_{2} \max$ pode ser definida como sendo a velocidade ou potência na qual o $\mathrm{VO}_{2}$ max é atingido durante um teste de cargas incrementais. Sendo assim, a $\mathrm{IVO}_{2} \max$ é o índice que melhor descreve a associação entre a potência aeróbia máxima $\left(\mathrm{VO}_{2} \max \right)$ e a $\mathrm{EM}^{1}$.

Embora esses índices tenham sido amplamente estudados, existem poucas evidências científicas para se formular recomendações de treinamento, devido ao número limitado de pesquisas envolvendo treinamento e pelas diferentes limitações que elas apresentam. Mesmo que essas evidências científicas sejam limitadas, ainda é possível que importantes recomendações para o treinamento possam ser formuladas, integrando informações derivadas dos estudos que envolveram treinamento com outros conhecimentos científicos. Este conhecimento pode incluir as respostas fisiológicas agudas em diferentes intensidades de exercício, as estruturas e processos que limitam os determinantes fisiológicos do rendimento aeróbio,e as adaptações associadas com suas melhoras.

Deste modo, este capítulo tem como objetivo trazer informações atuais e relevantes a respeito dos aspectos fisiológicos determinantes do rendimento e treinamento dos esportes aeróbios, através de uma linguagem clara e simples, proporcionando informações que podem ser bastante proveitosas tanto para atletas e técnicos quanto para pesquisadores e estudiosos da área esportiva. Primeiramente, serão abordados os principais aspectos bioenergéticos envolvidos no metabolismo aeróbio e posteriormente, serão discutidos os principais índices fisiológicos relacionados ao rendimento aeróbio.

\section{Aspectos bioenergéticos}

Durante o exercício, a demanda energética do músculo esquelético aumenta consumindo uma quantidade maior de trifosfato de adenosina (ATP). No entanto, os estoques de ATP são bem limitados, significando que a produção de ATP deve ocorrer na mesma velocidade na qual ele é utilizado, para que o exercício possa continuar por tempo prolongado. Existem três processos distintos e integrados que operam para satisfazer a demanda energética do músculo. $\mathrm{O}$ sistema anaeróbio que pode ser dividido em alático e lático. O sistema alático compreende a quebra da creatina fosfato (CP) e as moléculas de ATP já presentes dentro do músculo. O sistema lático refere-se à combustão parcial da glicose ou glicogênio. A quebra destas duas moléculas irá gerar ácido lático com a sua imediata conversão para lactato. E finalmente, o sistema aeróbio que se refere à combustão completa dos carboidratos (glicose e glicogênio), gorduras e em alguns casos proteínas na presença do oxigênio $\left(\mathrm{O}_{2}\right)$.

No início de um exercício de baixa intensidade, os sistemas anaeróbios alático e lático contribuem com a significante proporção na ressíntese de ATP até que uma estabilidade seja alcançada pelo metabolismo aeróbio. O retardo de tempo (1-2 min), até que o sistema aeróbio seja capaz de atender ou se aproximar da demanda energética, é devido ao aumento gradual do fluxo 
sanguíneo (oferta de oxigênio) e da ativação das suas várias reações enzimáticas ${ }^{2}$. Durante exercícios de alta intensidade, a demanda de ATP pela contração é muito alta, uma estabilidade nunca é alcançada e a fadiga muscular ocorre rapidamente. Nestas circunstâncias, a ressíntese do ATP derivado do sistema anaeróbio normalmente conta com a maior contribuição para o total de ATP ressintetizado 3 .

Recentemente, tem sido demonstrado que o sistema aeróbio responde surpreendentemente rápido a demanda energética ao início do exercício, tendo um importante papel também durante exercícios de alta intensidade. Em média, um exercício realizado de forma máxima, com duração em torno de 75s, parece utilizar, aproximadamente, igual energia dos sistemas aeróbio e anaeróbio ${ }^{4}$. No entanto, este tempo não seria fixo, mas provavelmente dependente do estado (sedentários vs. atletas) e especificidade (velocidade vs. resistência) do treinamento. Apesar disso, este ainda é um tempo consideravelmente mais curto do que tem sido tradicionalmente sugerido em alguns livros clássicos de fisiologia do exercício ${ }^{5,6}$. Portanto, distâncias a partir dos $200 \mathrm{~m}$ na natação e $800 \mathrm{~m}$ para o atletismo, já teriam um predomínio do metabolismo aeróbio no suprimento total da energia necessária.

É importante ressaltar que todos os sistemas são "acionados" no início do exercício, mas como eles têm grandes diferenças na quantidade total de energia disponível (capacidade) e na velocidade de produção energética (potência), fica óbvio que a relativa contribuição dos sistemas energéticos para um dado esforço, vai depender da sua intensidade e duração? Além disso, não há dúvidas de que cada sistema seja mais capacitado para proporcionar energia para um diferente tipo de evento ou atividade, no entanto, isto não quer dizer que ocorra alguma exclusividade, isto é, a ausência total de qualquer um dos sistemas energéticos. Assim, os sistemas energéticos contribuem sequencialmente sem o "desligamento" de qualquer um deles, mas em uma característica de superposição para atender à demanda energética do exercício ${ }^{4}$.

\section{Possíveis mecanismos de fadiga para eventos aeróbios}

De todos os possíveis aspectos relacionados com a fadiga, o acúmulo de lactato e íons $\mathrm{H}^{+}$ provenientes do metabolismo anaeróbio, são com certeza os mais difundidos, seja pela litera- tura específica ou pelo meio esportivo (atletas e técnicos) como principais causadores da fadiga muscular. No entanto, essa visão que o acúmulo de lactato e íons $\mathrm{H}^{+}$teriam um efeito negativo sobre o rendimento está sendo desafiada por recentes achados, utilizando procedimentos experimentais em fibras musculares isoladas em laboratório ${ }^{8,9}$. Estes estudos têm demonstrado que o acúmulo destes metabólitos pode aumentar a força de contração, diminuindo a inibição no processo de excitação-contração causado, provavelmente, pelo acúmulo de $\mathrm{K}^{+}$extracelular, isto é, quando o ácido lático foi infundido na fibra fatigada contribuiu para que esta voltasse a responder aos estímulos elétricos ${ }^{8,9}$. No entanto, uma falha na ativação muscular pode ser facilmente demonstrada durante contrações induzidas eletricamente em laboratório, ao contrário do exercício voluntário no qual este comportamento não tem sido comumente observado. Desta forma, muitos pesquisadores têm questionado estes novos achados, sugerindo que eles seriam inicialmente válidos somente para as condições experimentais usadas, não sendo possível uma transferência desses achados para condições normais de exercício, utilizando grandes grupos musculares como, por exemplo, a corrida $^{10-12}$. Seria, portanto, prematuro eliminar o acúmulo de íons $\mathrm{H}^{+}$como uma potencial causa da fadiga em algumas circunstâncias.

Apesar dos aspectos descritos anteriormente, o lactato, por si só, não pode mais ser considerado como um usual suspeito pelos "crimes" metabólicos, mas sim, como uma peça chave no metabolismo celular, regional e corporal. Parte da energia necessária para o movimento é produzida através da degradação da glicose e os produtos gerados, piruvato ou lactato, são os principais substratos utilizados posteriormente no processo de oxidação completa da glicose. Ainda dentro da fibra muscular, o lactato pode ser reconvertido a piruvato para ser oxidado na mitocôndria. Da mesma forma, ele pode ser transportado para fibras adjacentes, ou até a corrente sanguínea, através da qual ele pode alcançar diversos tecidos (outras musculaturas, fígado, coração) que podem utilizar esse lactato para produzir energia aerobiamente ou para repor os estoques de glicogênio ${ }^{13}$. É através desse mecanismo que, durante o exercício, fibras musculares inativas podem "doar energia" para musculatura que está sendo mais solicitada. Essas fibras inativas produzem lactato, que 
entra na corrente sanguínea, é transportado até a musculatura ativa e então utilizado para produzir energia. $\mathrm{O}$ consumo do lactato proveniente de outras fibras musculares poupa as reservas energéticas da musculatura que está sendo mais solicitada, prolongando o tempo de exercício. Estes aspectos ocorrem em situações que a intensidade de exercício, e conseqüentemente, a produção de lactato, não são muito elevadas. Por exemplo, durante intensidades de exercício até a chamada máxima fase estável de lactato, uma concentração modesta de lactato (na média, em torno de $4 \mathrm{mM}$ ) seria, provavelmente, vantajosa pela possibilidade de distribuir substratos energéticos para os diferentes tecidos. No entanto, durante exercícios mais intensos os níveis de lactato sanguíneo podem atingir valores em torno de $20 \mathrm{mM}$, com valores musculares ainda mais extremos. É provável que sobre estas condições de concentrações de lactato elevadas, o aumento da acidez causaria dor e desconforto, além de todos os aspectos deletérios associados à fadiga.

Os aspectos que foram discutidos anteriormente estariam associados aos prováveis mecanismos da fadiga para eventos com duração de até, aproximadamente, 1 hora. Mais adiante, serão abordados os prováveis mecanismos responsáveis pelo término do exercício de longa duração (mais de uma hora). Para estas durações, a depleção do glicogênio muscular e hepático e fatores relacionados com a termorregulação corporal também podem ser importantes para o rendimento máximo. Enquanto exercícios de alta intensidade $\left(150 \% \mathrm{VO}_{2} \max \right)$ quando realizados de forma intermitente podem reduzir muito os valores de glicogênio muscular em apenas $30 \mathrm{~min}^{14}$. Os exercícios contínuos sustentados por períodos prolongados de tempo somente acarretarão uma profunda redução no glicogênio muscular quando realizados abaixo de $90 \%$ do $\mathrm{VO}_{2}$ max e conseqüentemente, com uma duração provavelmente maior que 90 min. Portanto, se não realizado de forma intermitente, seja com pausa passiva ou com grande variação de intensidades, um exercício realizado abaixo de aproximadamente $90 \mathrm{~min}$, provavelmente, será limitado principalmente pelo acúmulo de metabólitos.

Em relação ao glicogênio muscular, quanto maior a intensidade do exercício maior será a utilização de glicogênio muscular. A maneira mais efetiva de "economizar" glicogênio mus- cular é aumentando a utilização das gorduras como substrato energético ${ }^{15}$. No entanto, um recente estudo demonstrou em exercícios de baixa intensidade $\left(63 \% \mathrm{VO}_{2} \max \right)$ com duração bem prolongada (180 min), que a suplementação de carboidratos pode aumentar a taxa de oxidação de glicose e poupar glicogênio muscular ${ }^{16}$. Existem duas formas de aumentar a utilização de gorduras como fonte energética. Para uma mesma intensidade de exercício, a única maneira é através do treinamento aeróbio, que é capaz de aumentar os estoques de gorduras dentro da fibra muscular e a velocidade das reações, envolvendo a quebra desta gordura estocada. Outro modo seria diminuindo a intensidade do exercício, fazendo com que os processos responsáveis pela oxidação das gorduras consigam contribuir com uma maior fração da energia total requerida. Vale ressaltar, por exemplo, que corredores de elite correm uma maratona em velocidades que variam de $80-90 \% \mathrm{VO}_{2}$ max e completam a prova entre 2 horas e $5 \mathrm{~min}$ - 2 horas e $15 \mathrm{~min}$. Embora esses atletas sejam altamente adaptados a usar gordura como combustível e devem fazer isso durante os treinamentos, eles competem a uma intensidade tão elevada (para esta duração) que a grande adaptação em estocar e oxidar carboidratos, podem ser suficientes para suprir as altas velocidades de corrida. No entanto, nesse nível de rendimento, muitas vezes diferenças mínimas separam o primeiro do segundo colocado e neste caso, mesmo tendo pouca influência, uma maior utilização de gorduras durante o exercício pode fazer a diferença. Em provas com durações maiores que a maratona, a capacidade de oxidar grandes quantidades de gordura passa a ser de extrema importância e decisiva para o rendimento máximo ${ }^{17}$.

Em relação ao glicogênio hepático, este nunca atingiria níveis críticos se durante o exercício prolongado for realizada uma correta suplementação com carboidratos, uma vez que a função do glicogênio hepático é manter os níveis de glicose sanguínea adequada para o funcionamento de outros órgãos (principalmente o cérebro), além da musculatura que está sendo exercitada. Portanto, uma suplementação externa de glicose consegue suprir tranquilamente essa demanda. Caso essa reposição não seja realizada, os estoques de glicogênio hepático durariam em média de 150 a 210 min, dependendo da intensidade do exercício. Além disso, se a intensidade de exercício for menor que 70\% 
$\mathrm{VO}_{2}$ max a depleção do glicogênio hepático poderá ocorrer antes da depleção do glicogênio muscular, fazendo com que ocorra o término do exercício devido a hipoglicemia ${ }^{18}$.

\section{Índices fisiológicos relacionados \\ ao rendimento aeróbio}

$\mathrm{O}$ consumo máximo de oxigênio $\left(\mathrm{VO}_{2} \max \right)$ tem sido um índice muito utilizado nas últimas décadas para avaliação do nível de aptidão aeróbia, seja de atletas ou sedentários, por estar relacionado com capacidades metabólicas e cardiovasculares. Este é o índice fisiológico que melhor representa a potência aeróbia máxima. Em outras palavras, é uma medida da quantidade máxima de energia que pode ser produzida pelo metabolismo aeróbio em uma determinada unidade de tempo. Na prática, é um índice que, em parte, parece explicar o melhor desempenho apresentado por atletas de resistência, demonstrado por valores de $\mathrm{VO}_{2}$ max até duas vezes maiores do que em indivíduos sedentários ${ }^{19}$. Fica bem claro que para um bom desempenho em esportes de resistência é necessário um elevado valor de $\mathrm{VO}_{2}$ max, mas não obrigatoriamente os atletas que possuem os valores mais altos deste índice, são os de melhor desempenho aeróbio ${ }^{20}$. Observando esta única variável, fica difícil explicar a grande variação de rendimento que ocorre em atletas de alto nível com elevado valor de $\mathrm{VO}_{2}$ max. Devido a essa "falha" do $\mathrm{VO}_{2}$ max em explicar essas diferenças, índices associados à resposta do lactato sanguíneo ao exercício, a economia de movimento (EM) e intensidade correspondente ao $\mathrm{VO}_{2} \max \left(\mathrm{IVO}_{2} \max \right)$, têm sido explorados nos últimos anos ${ }^{20-22}$.

O lactato como marcador fisiológico tem sido usado como uma importante ferramenta para controlar a intensidade do exercício aeróbio. Como discutido anteriormente, não é que a molécula de lactato por si só cause fadiga, mas o seu acúmulo no sangue reflete um aumento do metabolismo anaeróbio e/ou uma diminuição nos mecanismos responsáveis pela remoção do lactato, ocasionando a perda de um estado de equilíbrio no organismo. Embora diferentes terminologias e critérios venham sendo empregados para a identificação dos índices associados à resposta do lactato sanguíneo durante o exercício submáximo, basicamente duas intensidades de exercício têm sido determinadas:

Intensidade imediatamente anterior ao aumento do lactato sanguíneo em relação aos valores de repouso, durante um exercício de cargas crescentes, comumente chamadas de limiar de lactato (LL) ou limiar aeróbio ${ }^{23}$. Neste critério, normalmente, não se empregam concentrações fixas de lactato, encontrando-se entre 1,5 e $3 \mathrm{mM}$, com a intensidade de esforço correspondendo entre 40 e $80 \% \mathrm{VO}_{2} \max$, dependendo do nível de aptidão aeróbia do sujeito (Figura 1). Em intensidades acima do LL, o lactato sanguíneo ainda se mantém estável, mas em concentrações acima dos valores de repouso (Figura 2, $14 \mathrm{~km} / \mathrm{h}$ ).

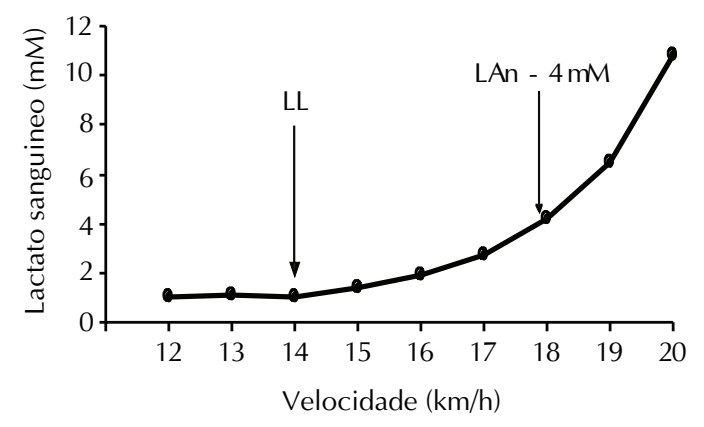

Figura 1. Comportamento do lactato sanguíneo durante um teste de cargas incrementais para determinação do limiar de lactato (LL) e do limiar anaeróbio (LAn) durante a corrida.

Intensidade de máxima fase estável de lactato sanguíneo (MFEL) é a maior intensidade de exercício na qual o lactato produzido e liberado pelos músculos para a corrente sanguínea é semelhante à taxa na qual ele é removido do sangue, existindo ainda um equilíbrio na concentração de lactato ao longo do exercício ${ }^{24}$. A máxima fase estável de lactato parece representar não só um equilíbrio da concentração de lactato sanguíneo, mas também de outros parâmetros fisiológicos ${ }^{25}$. É determinada individualmente através da realização de 3-5 repetições de carga constante, em diferentes dias, com 30 min de duração cada uma (Figura 2). Para tentar retirar essa desvantagem do grande número de testes, Heck et a ${ }^{126}$ propuseram a identificação indireta da MFEL com base em um único protocolo contínuo de cargas progressivas, adotando uma concentrarão fixa de $4 \mathrm{mM}$. Este protocolo vem sendo comumente chamado de limiar anaeróbio (LAn) (Figura 1). Infelizmente, o LAn vem sendo erroneamente interpretado. Esse protocolo não permite retirar grandes informações sobre o metabolismo anaeróbio, assumindo que a partir desse ponto o predomino passa a ser anaeróbio, mais propriamente ele também tenta refletir o 
balanço entre a "entrada" e a "saída" do lactato no sangue. Ambos os métodos, encontram-se em uma zona de intensidade correspondente a $70-92 \% \mathrm{VO}_{2}$ max, com as variações também sendo dependentes do estado de condicionamento físico.

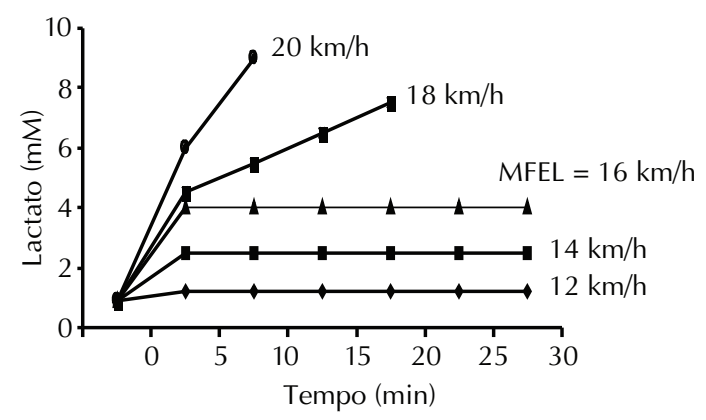

Figura 2. Protocolo de determinação da máxima fase estável de lactato (MFEL) sanguíneo durante a corrida.

Diferente do $\mathrm{VO}_{2} \max$, estes índices relacionados com a resposta do lactato sanguíneo são os que melhor representam a capacidade aeróbia, indicando teoricamente a quantidade total de energia que pode ser fornecida pelo metabolismo aeróbio e desta forma, demonstrando uma grande relação com o rendimento de longa duração. O LL, LAn e MFEL determinados a partir da resposta do lactato sanguíneo ao exercício, são capazes de selecionar os melhores e/ou os piores rendimentos aeróbios até mesmo em atletas de elite ${ }^{20}$.

A EM representa o custo de oxigênio para uma dada atividade submáxima. Alguns trabalhos têm demonstrado que a economia de movimento pode variar em até $15 \%$, mesmo em grupos de ciclistas bem treinados ${ }^{27}$ ou em corredores de elite ${ }^{28}$. Com base nestas variações da EM, pode-se entender porque a intensidade de exercício associada ao $\mathrm{VO}_{2} \max \left(\mathrm{IVO}_{2} \max \right)$ e o desempenho aeróbio podem ser bem diferentes entre atletas que possuem valores similares de $\mathrm{VO}_{2} \max$ (Figura 3). Genericamente, a $\mathrm{IVO}_{2}$ max pode ser definida como sendo a velocidade ou potência na qual $\mathrm{o} \mathrm{VO}_{2}$ max é atingido durante um teste de cargas incrementais até exaustão (estágios de 3 min na sua maioria). Para determinação direta do $\mathrm{VO}_{2} \max$ e $\mathrm{IVO}_{2} \max$ existe a necessidade de equipamentos de alto custo para mensuração do $\mathrm{VO}_{2}$, no entanto, medidas indiretas podem também ser obtidas através de teste incremental ${ }^{29}$ ou teste de carga constante com exaustão em torno de 6 min $^{22}$ sem a necessidade da medida direta do $\mathrm{VO}_{2}$.
Essa medidas indiretas da $\mathrm{IVO}_{2}$ max podem ser tranquilamente utilizadas como marcadores de desempenho aeróbio e para prescrição individualizada do treinamento.

$\mathrm{A} \mathrm{IVO}_{2}$ max é o índice que melhor descreve a associação entre a potência aeróbia máxima $\left(\mathrm{VO}_{2} \max \right)$ e a economia de movimento. Essa associação é exemplificada na Figura 3. Observe que para qualquer intensidade de exercício o indivíduo $\mathrm{B}$ possui um menor $\mathrm{VO}_{2}$, portanto, um menor gasto energético. Além disso, apesar de possuírem o mesmo valor de $\mathrm{VO}_{2}$ max, para o sujeito $B$ este valor é atingido em uma maior intensidade. Isso se dá pelo fato de que em um determinado momento o $\mathrm{VO}_{2}$ max passa a não responder ou responde de maneira bem reduzida aos estímulos do treinamento (mesmo que de alta intensidade). No entanto, outros índices como a EM e os limiares relacionados à resposta do lactato continuam a responder ao treinamento e se associar com a melhora do rendimento aeróbio.

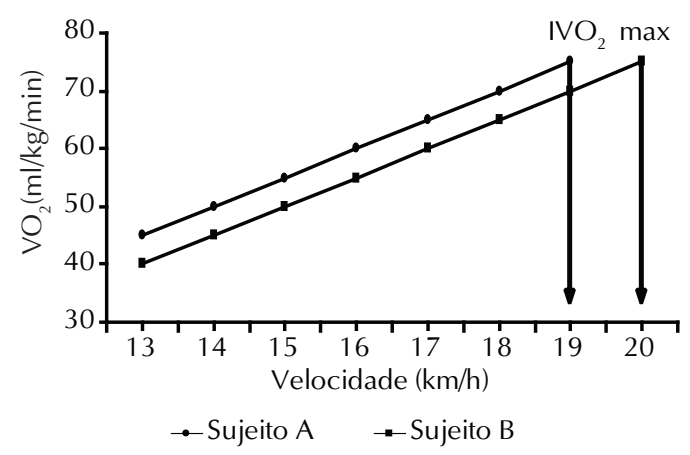

Figura 3. Relação entre a velocidade de corrida e o consumo de oxigênio $\left(\mathrm{VO}_{2}\right)$ em corredores com um mesmo valor de $\mathrm{VO}_{2}$ max durante um teste incremental (aumentos de $1 \mathrm{~km} / \mathrm{h}$ a cada $3 \mathrm{~min}$ ). Note que em função da diferença na economia de movimento eles apresentam diferentes velocidades associadas ao $\mathrm{VO}_{2} \max \left(\mathrm{VVO}_{2} \max \right)$.

Obviamente, em uma competição é melhor correr a 20 do que a $19 \mathrm{Km} / \mathrm{h}$. Olhando pelo lado do rendimento, melhor que ter um valor de $\mathrm{VO}_{2}$ max elevado é possuir esse valor em intensidades mais elevadas. No entanto, veremos com mais detalhes a seguir, que isso é válido para provas em intensidades próximas a da $\mathrm{IVO}_{2} \max$ (3-8 min). Porém, em algumas provas de maior duração, mais importante que ter um elevado valor de $\mathrm{IVO}_{2}$ max é conseguir se exercitar em porcentagens elevadas da $\mathrm{IVO}_{2}$ max ou $\mathrm{VO}_{2}$ max, e por conseqüência, possuir os índices relacionados à resposta do lactato (índices submáximos) também em uma alta $\% \mathrm{IVO}_{2} \max$. 
Dois estudos de caso podem demonstrar como alguns desses aspectos acontecem na prática. Um acompanhamento longitudinal da atual recordista mundial da Maratona, Paula Radcliff, no período de 1991-1995, demonstrou uma melhora substancial na EM de aproximadamente $9 \%$, refletida por uma redução no custo de oxigênio de 53 para $48 \mathrm{~mL} / \mathrm{kg} / \mathrm{min}$ para correr a uma velocidade de $16 \mathrm{~km} / \mathrm{h}^{30}$. Recentemente, foi também demonstrado que a eficiência mecânica melhorou $8 \%$ em um período de 7 anos de treinamento para um ciclista que se tornou o maior vencedor de todos os tempos do Tour de France ${ }^{31}$. Estes dados demonstram que a EM ou a eficiência no ciclismo são de fato sensíveis ao treinamento, com as melhoras ocorrendo a taxas de apenas 1-2\% ao ano. A partir dos Jogos Olímpicos de 1964 a dominação dos africanos, especialmente Quenianos, nas corridas de fundo e meio fundo torna-se um fenômeno inigualável em qualquer outro esporte $^{32}$. Dois estudos falharam em responder fisiologicamente o porquê da superioridade dos africanos nas corridas de distância, embora dois dos melhores corredores Quenianos tenham sido os mais eficientes até hoje estudados ${ }^{33,34}$. Com uma insignificante menor proporção de fibras do tipo I, a importante diferença foi que os corredores negros conseguem sustentar substancialmente maior proporção do seu $\mathrm{VO}_{2}$ max quando correm distâncias acima de $5 \mathrm{~km}$. Essa diferença se eleva ainda mais com o aumento da distância. Assim, o corredor negro parece ter uma superior resistência à fadiga, mas não uma maior potência aeróbia $\left(\mathrm{VO}_{2} \max \right)$.

$\mathrm{O}$ quanto cada índice fisiológico representa do rendimento total vai depender da duração da prova e, portanto, da intensidade relativa que pode ser mantida $\left(\% \mathrm{IVO}_{2} \max \right)$. A duração de um exercício e sua intensidade é o que caracteriza o tipo de sistema energético predominantemente utilizado. Este aspecto é fundamental quando se deseja elaborar um programa de treinamento visando à aplicação da sobrecarga (intensidade x volume), particularmente em relação aos seus aspectos metabólicos (potências e capacidades anaeróbia e aeróbia). Com base nessas considerações e no fato de que alguns exercícios podem apresentar participações percentuais importantes de duas vias metabólicas (aeróbio e anaeróbio), é possível tentar identificar em que durações de exercícios o $\mathrm{VO}_{2} \max$, a $\mathrm{IVO}_{2} \max$, e a resposta do lactato ao exercício, podem ser importantes para o rendimento e treinamento.

Para provas que possuem ainda uma grande contribuição (40\%) do metabolismo anaeróbio, como é o caso dos $800 \mathrm{~m}$ na corrida e $200 \mathrm{~m}$ na natação (duração em torno de 1,5 a 2 min.), o $\mathrm{VO}_{2}$ max apresenta uma relação moderada com o rendimento $(r=0,4-0,7)$, e que aumenta quando se relaciona com a $\mathrm{IVO}_{2} \max (r=0,8)^{21}$. Para a programação do treinamento nestas durações deveriam ser levados em consideração métodos de treinamento que desenvolvam principalmente a $\mathrm{IVO}_{2}$ max e também a capacidade anaeróbia lática. Nas provas realizadas entre 3-8 min. (por exemplo, 1.500-3.000 m no atletismo, $400-800 \mathrm{~m}$ na natação, $4.000 \mathrm{~m}$ no ciclismo), a participação anaeróbia, mesmo que reduzida (10 - 20\%), ainda pode ser considerada importante. Nestas condições, em que o exercício é executado entre $110 \%$ e $95 \%$ do $\mathrm{VO}_{2} \max$, tanto o $\mathrm{VO}_{2} \max$ como principalmente a $\mathrm{IVO}_{2}$ max apresentam uma elevada relação com o rendimento $(r=0,8-0,9)^{21,35}$. Nestas distâncias, os índices associados à resposta do lactato sanguíneo (capacidade aeróbia) parecem não contribuir de maneira significativa para o desempenho, não devendo ser o objetivo principal do treinamento. Portanto, os programas de treinamento para estas distâncias devem privilegiar a melhora da potência aeróbia, particularmente da $\mathrm{IVO}_{2}$ max. É importante destacar ainda que no período específico de treinamento, sessões que busquem desenvolver a capacidade anaeróbia lática e a capacidade de tolerar dor e desconforto provocados pela acidose também poderiam contribuir para a melhora do rendimento nessas distâncias.

Para durações maiores (acima dos $10 \mathrm{~min}$. até $40 \mathrm{~min}$.), nas quais a participação anaeróbia diminui sensivelmente, o exercício é realizado entre 85 e $95 \%$ do $\mathrm{VO}_{2}$ max. Embora os indivíduos possuam valores bem elevados de $\mathrm{VO}_{2}$ max, os níveis de relação deste índice com o desempenho diminui $(\mathrm{r}=0,6-0,7)$, isto quer dizer que os indivíduos com maior $\mathrm{VO}_{2} \max$ não serão necessariamente os de melhor desempenho ${ }^{21}$. Já para a intensidade associada com $\mathrm{VO}_{2} \max$ $\left(\mathrm{IVO}_{2} \max \right)$ a relação com o desempenho ainda continua elevada $(r=0,8-0,9)^{36}$. Para estas durações, a capacidade aeróbia começa a ter um papel decisivo, com os índices relacionados com a resposta do lactato (MFEL, LAn), apresentando também uma elevada relação com o 
rendimento $(r>0,9)^{37}$. Portanto, o treinamento para essas provas deveria buscar não só a melhora da $\mathrm{IVO}_{2} \max$, mas também da capacidade aeróbia (MFEL).

Em provas como meia-maratona e maratona, triathlon na distância short (750 m natação, 20 $\mathrm{km}$ ciclismo, $5 \mathrm{~km}$ corrida) e Olímpico (1.500 $\mathrm{m}$ natação, $40 \mathrm{~km}$ ciclismo, $10 \mathrm{~km}$ corrida), apresentando uma duração entre $1 \mathrm{~h}$ à $2 \mathrm{~h} 30$ min. e executados entre $70 \%$ e $90 \%$ do $\mathrm{VO}_{2} \max$, a participação anaeróbia pode ser considerada desprezível. Aqui também os atletas possuem altos valores de $\mathrm{VO}_{2}$ max, mas este índice tem uma relação ainda menor com o desempenho $(\mathrm{r}$ $=0,4-0,65)$. No entanto, a $\mathrm{IVO}_{2}$ max continua a apresentar uma relação razoável com o rendimento $^{36}$. $\mathrm{O}$ índice que melhor se relaciona com o desempenho nestas durações é a resposta do lactato ao exercício (capacidade aeróbia). Para o treinamento de modalidades nesta duração, ainda seria necessário o desenvolvimento da $\mathrm{IVO}_{2} \max$. No entanto, uma grande ênfase deveria ser dada aos treinos que melhorem a capacidade aeróbia, máxima fase estável de lactato sanguíneo e em menor extensão o limiar de lactato.

Para eventos de duração ainda mais prolongada (Ultramaratonas, Ironman, travessias em águas abertas), realizados abaixo de $60 \%$ do $\mathrm{VO}_{2} \max$, nenhum índice fisiológico consegue de maneira efetiva explicar as variações de rendimento nestas provas, embora os atletas que competem nesta modalidade apresentem valores elevados tanto de potência $\left(\mathrm{VO}_{2} \max \right)$ como de capacidade aeróbia (MFEL e LL). Nestas provas, maior capacidade de estocar glicogênio, maior utilização de gorduras como substrato energético e aspectos relacionados à termorregulação serão muito importantes para o rendimento máximo ${ }^{38}$. O treinamento, embora possa ser feito para desenvolver ambas a potência e capacidade aeróbia, deve ter como objetivo principal a melhora da capacidade de estocar glicogênio e aumentar a utilização de gordura como substrato energético ${ }^{17}$.

\section{CONSIDERAÇÕES FINAIS}

Diferente do que se tem sido comumente apresentado, o metabolismo aeróbio responde rapidamente a demanda energética, com um exercício máximo de $75 \mathrm{~s}$ apresentando iguais contribuições do sistema aeróbio e anaeróbio para a produção total de energia. No entanto, em exercícios acima dessa duração os possíveis mecanismos da fadiga relacionados ao metabolismo anaeróbio ainda são os principais determinantes da tolerância ao exercício. Em durações mais prolongadas (acima de uma hora), a depleção de glicogênio, o desequilíbrio hídrico e eletrolítico, elevação da temperatura corporal terão um papel decisivo no rendimento. Dependendo da duração da modalidade que se compete, os treinos deverão objetivar uma melhora da $\mathrm{IVO}_{2} \max$, capacidade anaeróbia lática e tolerância a acidose, para as competições mais curtas (abaixo de $10 \mathrm{~min}$ ), capacidade aeróbia para durações intermediárias (10-60 min), e a melhora da capacidade de estocar glicogênio e aumentar a utilização de gordura, nas modalidades muito prolongadas (acima de $60 \mathrm{~min}$ ).

\section{REFERÊNCIAS BIBLIOGRÁFICAS}

1. Caputo F, Stella SG, Mello MT, Denadai BS. Indexes of power and aerobic capacity obtained in cycle ergometry and treadmill running: comparisons between sedentary, runners, cyclist and triathetes. Rev Bras Med Esporte 2003;9 (4):231-37.

2. Grassi B. Regulation of oxygen consumption at exercise onset: is it really controversial? Exerc Sport Sci Rev 2001;29(3):134-8.

3. Bangsbo J, Gollnick PD, Graham TE, Juel C, Kiens B, Mizuno M, et al. Anaerobic energy production and $\mathrm{O} 2$ deficit-debt relationship during exhaustive exercise in humans. J Physiol 1990;422(1):539-59.

4. Gastin PB. Energy system interaction and relative contribution during maximal exercise. Sports Med 2001;31(10):725-41.

5. Fox EL, Bowers RW, Foss ML. The physiological basis for exercise and sport. Dubuque, Wm. C. Brown Communications, 1993.

6. Mcardle WD, Katch FI, Katch VL. Essentials of exercise physiology. 2 nd rev. ed. Philadelphia: Williams and Wilkins; 2000.

7. Spencer MR, Gastin PB. Energy system contribution during 200 to $1500 \mathrm{~m}$ running in highly trained athletes. Med Sci Sports Exerc 2001;33(1):157-62.

8. Nielsen OB, de Paoli F, and Overgaard K. Protective effects of lactic acid on force production in rat skeletal muscle. J Physiol 2001;536(1):161-6.

9. Pedersen TH, Nielsen OB, Lamb GD, and Stephenson DG. Intracellular acidosis enhances the excitability of working muscle. Science 2004;305(5687):1144-7.

10. Bangsbo J, Juel C. Counterpoint: lactic acid accumulation is a disadvantage during muscle activity. J Appl Physiol 2006;100(4):1412-3.

11. Sahlin K. Point:Counterpoint authors respond to commentaries on "Lactic acid accumulation is an advantage/disadvantage during muscle activity". J Appl Physiol 2006;101(1):367. 
12. Gladden LB, Hogan MC. Lactic acid accumulation is an advantage/disadvantage during muscle activity. J Appl Physiol 2006;100(6):2100-1.

13. Gladden LB. Lactate metabolism: a new paradigm for the third millennium. J Physiol 2004;558(1):5-30.

14. Astrand PO, Rodahl K. Textbook of Work Physiology. 2 nd rev. ed. New York, McGraw Hill, 1977.

15. Achten J, Jeukendrup AE. Optimizing fat oxidation through exercise and diet. Nutrition 2004;20(78):716-27.

16. Stellingwerff T, Boon H, Gijsen AP, Stegen JH, Kuipers H, van Loon LJ. Carbohydrate supplementation during prolonged cycling exercise spares muscle glycogen but does not affect intramyocellular lipid use. Pflugers Arch 2007;454(4):635-47.

17. Noakes TD. Physiological models to understand exercise fatigue and the adaptations that predict or enhance athletic performance. Scand J Med Sci Sports 2000;10 (3):123-45.

18. Noakes TD. Lore of running. Discover the science and spirit of running. $3 \mathrm{rd}$ ed. Champaign: Human Kinetics; 1991.

19. Caputo F, Denadai BS. Effects of aerobic endurance training status and specificity on oxygen uptake kinetics during maximal exercise. Eur J Appl Physiol 2004;93(1-2):87-95

20. Coyle EF. Integration of the physiological factors determining endurance performance ability. Exerc Sport Sci Rev 1995;23:25-63.

21. Brandon LJ. Physiological factors associated with middle distance running performance. Sports Med 1995;19(4):268-77.

22. Bosquet L, Léger L, Legros P. Methods to Determine Aerobic Endurance. Sports Med 2002;32(11):675700.

23. Yoshida T, Chida M, Ichioka M, Suda Y. Blood lactate parameters related to aerobic capacity and endurance performance. Eur J Appl Physiol 1987;56(1):7-11.

24. Beneke R. Methodological aspects of maximal lactate steady state-implications for performance testing. Eur J Appl Physiol 2003;89(1):95-9.

25. Baron B, Dekerle J, Robin S, Neviere R, Dupont L, Matran R, et al. Maximal lactate steady state does not correspond to a complete physiological steady state. Int J Sports Med 2003;24(8):582-7.

26. Heck H, Mader A, Hess G, Mucke S, Muller R, Hollmann W. Justification of the $4 \mathrm{mmol} / \mathrm{l}$ lactate threshold. Int J Sports Med 1985;6(3):117-30.

27. Coyle EF, Feltner ME, Kautz SA, Hamilton MT, Montain SJ, Baylor AM, et al. Physiological and biomechanical factors associated with elite endurance cycling performance. Med Sci Sports Exerc 1991;23(1):93-107.
28. Morgan DW, Martin PE, Krahenbuhl GS, Baldini FD. Variability in running economy and mechanics among trained male runners. Med Sci Sports Exerc 1991;23(3):378-83.

29. Machado CEP, Caputo F, Denadai BS. Intensidade de exercício correspondente ao VO2max durante o ciclismo: análise de diferentes critérios em indivíduos treinados. Rev Bras Educ Fís Esporte 2004;18(4):333-41.

30. Coyle EF. Physiological Regulation of Marathon Performance. Sports Med 2007;37(4-5):306-11.

31. Coyle EF. Improved muscular efficiency displayed as 'Tour de France' champion matures. J Appl Physiol 2005;98(6):2191-6.

32. Bale J, Sang J. Kenyan running. London, Frank Cass, 1996

33. Saltin B, Larsen H, Terrados N, Bangsbo J, Bak T, $\mathrm{Kim} \mathrm{CK}$, et al. Aerobic exercise capacity at sea level and at altitude in Kenyan boys, junior and senior runners compared with Scandinavian runners. Scand J Med Sci Sports 1995;5(4):209-21.

34. Saltin B. Adaptive responses to training at medium altitude; with a note on Kenyan runners and a proposal for a multi-centre study. Revised and extended version of Saltin B. Exercise and the Environment: focus on altitude. Res Quart Exerc Sport 1996;67(3):1-10.

35. Caputo F, Lucas RD, Mancine EC, Denadai BS. Comparação de diferentes índices obtidos em testes de campo para predição da performance aeróbia de curta duração no ciclismo. Rev Bras Ciên Mov 2001;9(4):13-7.

36. Noakes TD, Myburgh KH, Schall R. Peak treadmill running velocity during the VO2max test predicts running performance. J Sports Sci 1990;8(1):35-45.

37. Machado CEP, Caputo F, Lucas RD, Denadai BS. Fatores fisiológicos e antropométricos associados com a performance em subida no ciclismo off road. Rev Bras Ciên Mov 2002;10(4):35-40.

38. O’Toole ML, Douglas PS, Hiller WD. Applied physiology of a triathlon. Sports Med 1989;8(4):201-25.

\section{Endereço para correspondência \\ Fabrizio Caputo \\ UDESC}

Centro de Ciências da Saúde e do Esporte

Rua Pascoal Simone 358, Coqueiros 88080-350. Florianópolis, SC, Brasil E-mail: fabriziocaputo@hotmail.com 